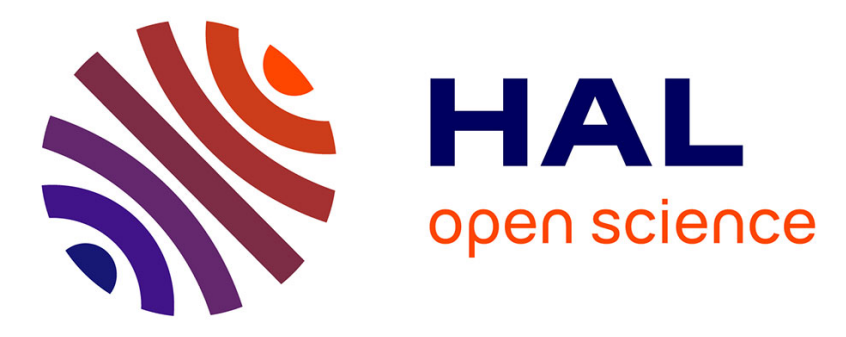

\title{
Simulation of skin effect via separated representations
} M. Pineda-Sanchez, Francisco Chinesta, J. Roger-Folch, M. Riera-Guasp, J. Pérez-Cruz, F. Daïm

\section{To cite this version:}

M. Pineda-Sanchez, Francisco Chinesta, J. Roger-Folch, M. Riera-Guasp, J. Pérez-Cruz, et al.. Simulation of skin effect via separated representations. COMPEL: The International Journal for Computation and Mathematics in Electrical and Electronic Engineering, 2010, 29 (4), pp.919-929. 10.1108/03321641011044334. hal-01008914

\section{HAL Id: hal-01008914 https://hal.science/hal-01008914}

Submitted on 29 Jan 2017

HAL is a multi-disciplinary open access archive for the deposit and dissemination of scientific research documents, whether they are published or not. The documents may come from teaching and research institutions in France or abroad, or from public or private research centers.
L'archive ouverte pluridisciplinaire HAL, est destinée au dépôt et à la diffusion de documents scientifiques de niveau recherche, publiés ou non, émanant des établissements d'enseignement et de recherche français ou étrangers, des laboratoires publics ou privés. 


\title{
Simulation of skin effect via separated representations
}

\author{
M. Pineda Sanchez \\ Department of Electrical Engineering, Universidad Politécnica de Valencia, \\ Valencia, Spain \\ F. Chinesta \\ GEM UMR CNRS École Centrale de Nantes, Nantes, France \\ J. Roger Folch, M. Riera Guasp and J. Pérez Cruz \\ Department of Electrical Engineering, Universidad Politécnica de Valencia, \\ Valencia, Spain \\ F. Daïm \\ École Nationale Supérieure des Mines de Paris, \\ Centre des Matériaux P.M. Fourt, Evry, France
}

\begin{abstract}
Purpose The purpose of this paper is to apply the method of separation of variables to obtain the current distribution in the slot of an electrical machine, taking into account the skin effect.

Design/methodology/approach A slot in an electrical machine, filled with a solid conductor, and fed with an externally imposed density current, presents a current distribution that depends on the skin effect. The magnetic potential vector is formulated and solved using a separate representation as a finite sum of unidimensional (space and time) functions, taking into account the boundary conditions. The proposed method obtains the transient and permanent distribution of the current in the interior of the slot, both in transient and steady regime, and the results at the end of the transient are compared with the analytic ones in permanent regime.
\end{abstract}

Findings The magnetic potential vector in the interior of a slot filled with a solid conductor can be expressed as a finite sum of just 16 modes, which capture the evolution of the field during the transient and permanent regime. These modes are expressed as the product of space and time functions, which have been obtained automatically by the separation of variables algorithm. Instead of solving multiple field problems, one for each time instant, the proposed method just solves two single variable differential equations, one in the time domain and other in the spatial one.

Research limitations/implications The application of the proposed method to non sinusoidal currents, such as those generated by variable speed drives, would allow to compute the field taking into account both the very small time scale of the pulse width modulation pulses, in the range of $\mathrm{kiloHz}$, and the wide time scale of the currents envelope, in the range of $0100 \mathrm{~Hz}$. Extension to 2D and 3D spatial configurations is also under consideration.

Originality/value Using the method of separation of variables to solve electromagnetic problems provides a new method which can simplify and speed up the computation of transient fields in multidimensional time and space domains.

Keywords Modelling, Computer applications, Electric current, Electromagnetism, Transient voltages, Simulation 


\section{Introduction}

Separated representations based on finite sums can help to reduce drastically the computer resources and the calculation costs that the functional approximation requires in multidimensional problems (Ammar et al., 2006, 2007, n.d.; Chinesta et al., 2008; Gonzalez et al., 2010). For example, the simulation of transient currents in motor slots exhibits an exponential growing, characteristic of mesh-based discretization strategies, as we introduce new dimensions (either spatial one or the time) in the model. Using the method of separation of variables, on the contrary, the number of degrees of freedom scales just linearly with the dimension of the space in which the model is defined. This method, also known as finite sums decomposition or proper generalized decomposition, expresses an $N$-dimensional function $u\left(x_{1}, x_{2}, \ldots, x_{N}\right)$ as:

$$
u\left(x_{1}, x_{2}, \ldots, x_{N}\right) \approx \sum_{i=1}^{i r} F_{1}^{i}\left(x_{1}\right) \cdot F_{2}^{i}\left(x_{2}\right) \cdots F_{N}^{i}\left(x_{N}\right)
$$

where the dimensions can be either spatial ones $(x, y, z)$, time, etc. If function $u$ is the unknown field involved in a partial differential equation (PDE), expressing it as equation (1) can reduce drastically the size of the mesh required to obtain it: instead of $N$-dimensional mesh, the problem can be solved with $N$ meshes of a much lower size. The problem with this approach is to be able to obtain the decomposition of the unknown field with an easy to apply numerical procedure. This procedure exists (Chinesta et al., 2008) and it is presented here applied to the problem of computing the current distribution inside of a machine slot with an externally imposed current distribution. This problem is solved in a 2D domain (one spatial dimension and time), but it can readily extended to a $3 \mathrm{D}$ or even to a $4 \mathrm{D}(x, y, z, t)$ domain.

\section{Analytical solution of the current in a rectangular slot in permanent regime}

The method of separation of variables is used to obtain the total current in the slot of an electrical machine filled with a solid copper conductor, fed with an externally imposed density current (Figure 1). First, the analytical solution in permanent regime will be obtained. Second, the transient solution will be obtained with the proposed method, and compared with the analytical solution once reached the permanent regime.

The slot has dimensions $b \times L$ and it is occupied by a material with constant properties $\mu \quad 4 \pi 10{ }^{7}$ Henries $/ \mathrm{m}$ and $\sigma \quad 59 \times 10^{6}$ Siemens $/ \mathrm{m}$. The slot has a total depth of $L \quad 30 \mathrm{~mm}$. An external density current $\mathbf{J}_{\mathbf{0}} \quad J_{0 \max } \cos (\omega \mathrm{t})$ is imposed in the conductor, in the $z$ direction, with $\omega \quad 2 \pi 50 \mathrm{rad} / \mathrm{s}$ and $J_{0 \max } \quad 10^{4} \mathrm{~A} / \mathrm{m}^{2}$. The magnetic field $\mathbf{H}$ and the magnetic flux density $\mathbf{B}$ verify:

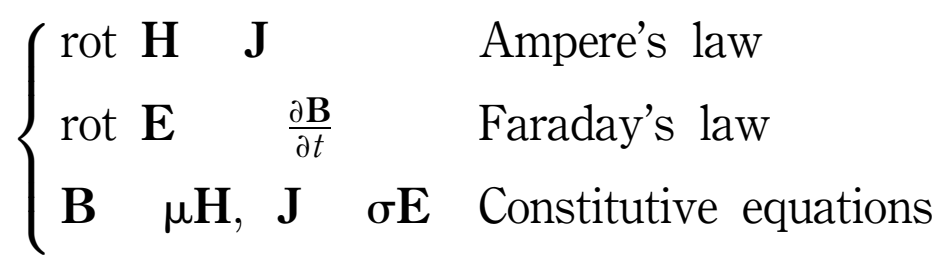

As all the currents have only z-component, so does the magnetic vector potential (MVP). The total current density in the conductor will be the sum of the externally imposed one and the induced current due to the time-variation of the magnetic flux density: 


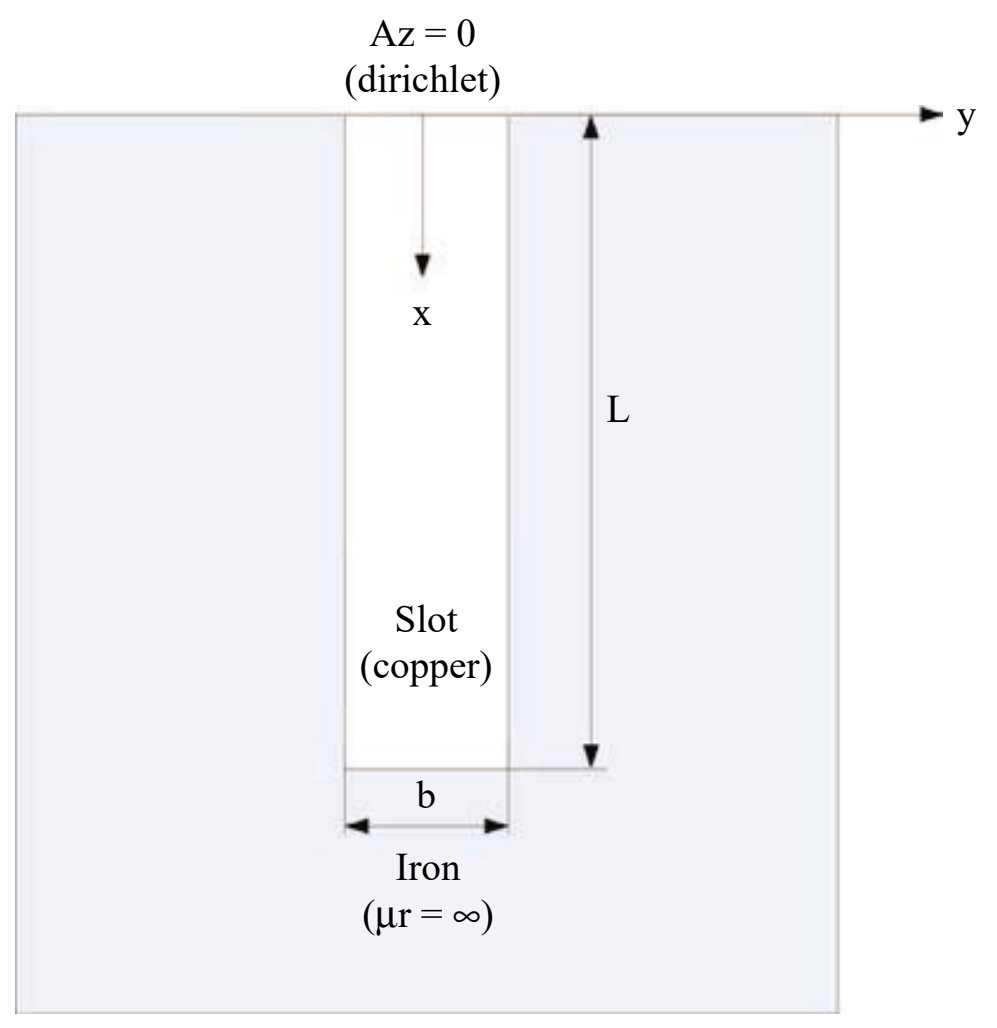

Figure 1.

Slot with a solid copper

conductor

$$
\begin{array}{lll}
\mathbf{J} & \mathbf{J}_{\text {ext }}+\mathbf{J}_{\text {ind }} & \mathbf{J}_{\text {ext }}+\sigma \mathbf{E}_{\text {ind }}
\end{array}
$$

Using the MVP A, so that $\mathbf{B} \operatorname{rot}(\mathbf{A})$, Faraday's law gives:

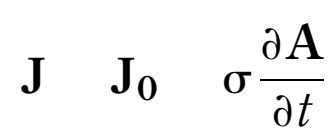

Both the currents and the MVP have only a $z$-components. Besides, as it is assumed infinite magnetic permeability of the iron (magnetic flux density normal to the walls), and due to the symmetry of the problem, this component has no variation in the $y$ direction, so applying Ampère law to equation (4) gives:

$$
\frac{\partial^{2} A}{\partial x^{2}} \quad \mu \sigma \frac{\partial A}{\partial t} \quad \mu J_{0}
$$

Assuming that the all the magnitudes vary sinusoidally with time (permanent regime), equation (5) can be expressed by means of complex phasors as:

$$
\frac{\partial^{2} A}{\partial x^{2}} \quad j \omega \mu \sigma A \quad \mu J_{0}
$$

what constitutes the diffusion equation of the magnetic potential vector in the slot.

Assuming that the top of the slot is a flux line, and that the magnetic flux density at the bottom of the slot is null the following boundary conditions hold:

$$
\left.A(0) \quad 0 \quad \frac{\partial A}{\partial x}\right|_{x \quad L} 0
$$


Equation (7) is a second order inhomogeneous differential equation with constant coefficients, so:

$$
A \underbrace{C_{1} e^{(\sqrt{ } j \omega \mu \sigma) x}+C_{2} e^{(\sqrt{ } j \omega \mu \sigma) x}}_{\text {General Solution }}+\underbrace{\frac{J_{0}}{j \omega \sigma}}_{\text {Particular Solution }}
$$

The constants $C_{1}$ and $C_{2}$ are found applying to equation (9) the boundary conditions (8), which gives:

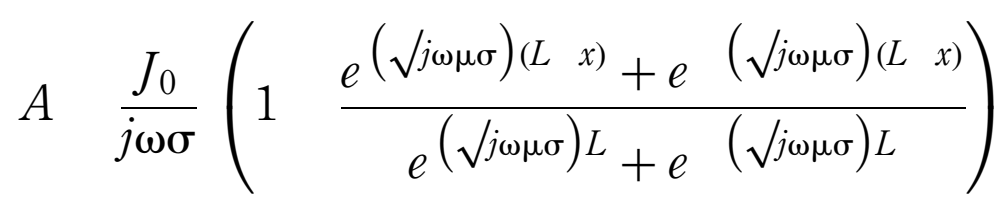

And, applying the definition of the depth of penetration factor $\delta$ (which only depends on the material properties and on the frequency):

$$
\begin{aligned}
& \delta \quad \sqrt{\frac{2}{\omega \mu \sigma}} \quad \sqrt{\frac{2}{2 \pi 50 \cdot 4 \pi 10^{7 \cdot 59.6 \cdot 10^{6}}}} \quad 9.21 \cdot 10^{3} \mathrm{~m}
\end{aligned}
$$

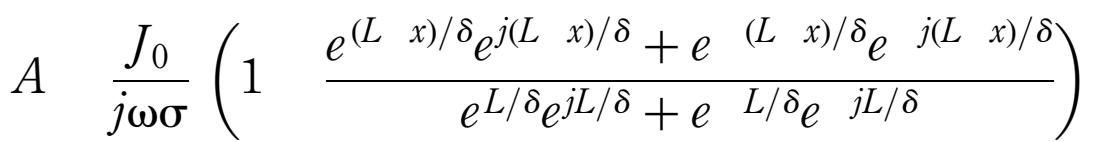

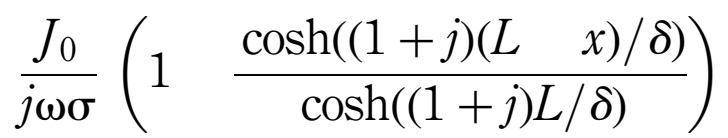

The current density in the slot is given by equation (6):

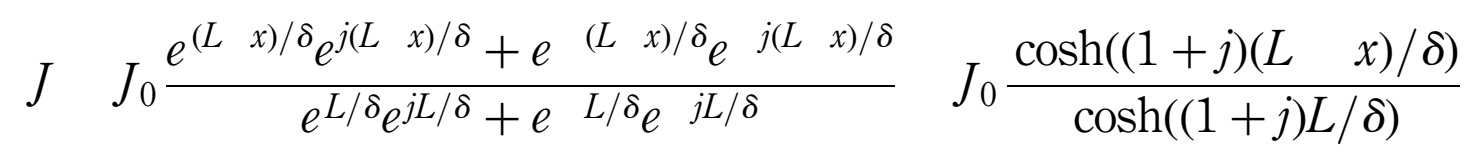

The variation of the magnitude and phase of equation (12) with the depth in the slot is shown in Figure 2.

Figure 3 shows the current in a phasor view, and also a 2D and 3D sinusoidal waves.
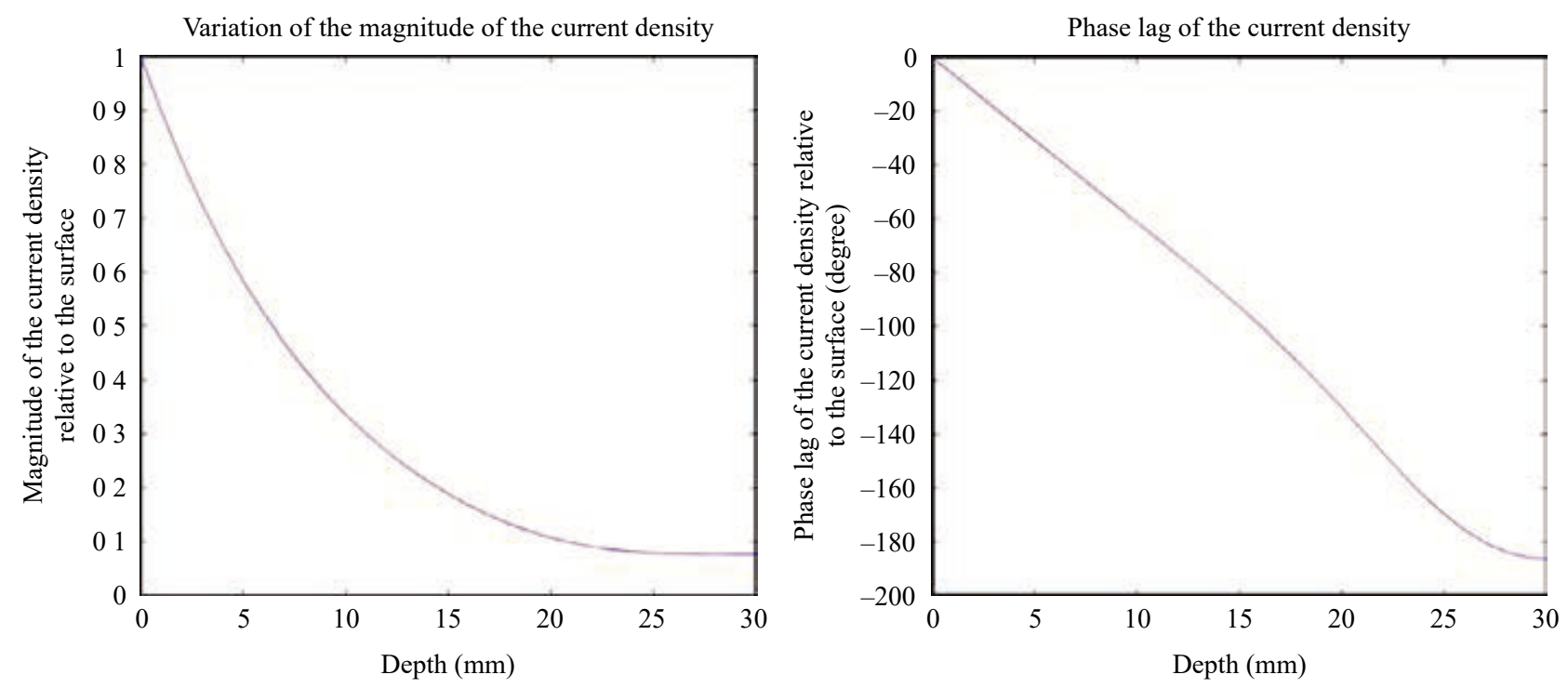

Figure 2. Variation of the magnitude of the current with the depth (left) and phase lag of the current (right), with respect to the current at the top of the slot 


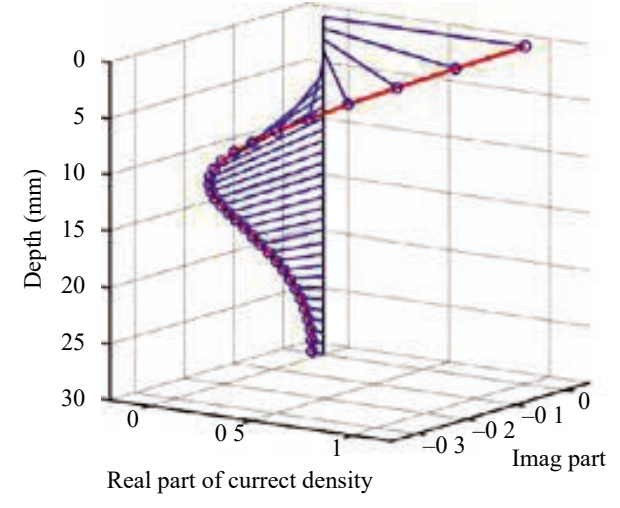

(a)

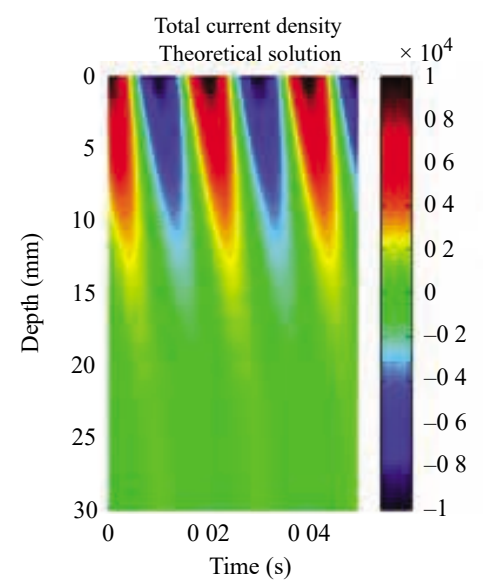

(b)

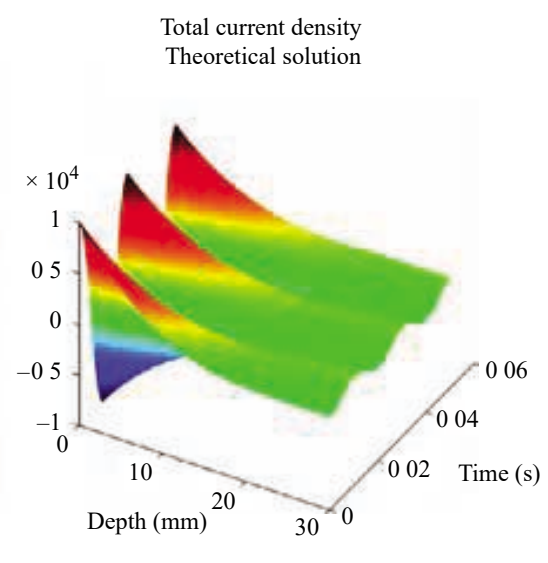

(c)

Notes: (a) Phasor view; (b) 2D view; (c) 3D view

Figure 3. Variation of the current density with depth, relative to the current at the top of the slot

\section{Transient solution of the current in a rectangular slot in transient regime}

In transient regime, the total current density in the slot $J$ can be found by solving equation (5), which is a $1 \mathrm{D}$ diffusion problem. In the case of homogeneous boundary conditions, the diffusion PDE is given by:

$$
\left\{\begin{array}{lll}
K \cdot \frac{\partial u(x, t)}{\partial t} \quad D \cdot \frac{\partial^{2} u(x, t)}{\partial x^{2}} \quad b(x, t) & \text { in } \Omega \quad(0, \mathrm{~L}), \mathrm{I} \quad(0, \mathrm{~T}) \\
u(0, t) \quad u(L, t) \quad 0 \text { on } \Gamma \equiv \partial \Omega & \text { Essential boundary conditions (EBC) } \\
u(x, 0) \text { at } t \quad 0 & \text { Initial conditions }
\end{array}\right.
$$

The field $u(x, t)$ and the second member $b(x, t)$ are represented as separate variables products:

$$
u(x, t) \quad \sum_{i=1}^{n} X_{i}(x) T_{i}(t) \quad b(x, t) \quad X_{b}(x) T_{b}(t)
$$

So equation (14) can be expressed (the independent variables are no longer shown for simplicity) as:

$$
K \cdot \sum_{i=1}^{n} X_{i} \frac{\partial T_{i}}{\partial t} \quad D \cdot \sum_{i=1}^{n} \frac{\partial^{2} X_{i}}{\partial x^{2}} T_{i} \quad X_{b} T_{b}
$$

Modes $X_{i}$ and $T_{i}$ can be computed numerically, using an iterative non-linear procedure. Suppose that the first $(n-1)$ modes are known. The $n$th mode is obtained via a Galerkin method using equation (13):

$$
\begin{aligned}
& u \quad \sum_{i=1}^{n} X_{i} T_{i}+X_{n} T_{n} \Rightarrow u^{*} \quad X_{n}^{*} T_{n}+X_{n} T_{n}^{*} \\
& \left.\int_{x}^{x}{ }_{0}^{L} \int_{t}^{t}{ }^{T} u^{*} \quad K \cdot \sum_{i=1}^{n} X_{i} \frac{\partial T_{i}}{\partial t} \quad D \cdot \sum_{i}^{n} \frac{\partial^{2} X_{i}}{\partial x^{2}} T_{i} \quad X_{b} T_{b}\right) \cdot d t \cdot d x \quad 0
\end{aligned}
$$


To find mode $n$ an iterative procedure is followed. Suppose that the mode $T_{n}$ is known at a given iteration, $\left(u^{*} \quad X_{n}^{*} \cdot T_{n}\right)$ so by substitution of equation (16) in equation (17) we get:

$$
\begin{aligned}
\int_{x}^{x}{ }^{x} X_{n}^{*}(K \sum_{i=1}^{n} \underbrace{\left(\int_{t}^{t}{ }^{T} T_{n} \frac{\partial T_{i}}{\partial t} d t\right)}_{\alpha_{i}} X_{i} & D \sum_{i}^{n} \underbrace{\left(\int_{t}^{t} T_{n}^{T} T_{n} T_{i} d t\right)}_{\beta_{i}} \frac{\partial^{2} X_{i}}{\partial x^{2}} \\
& \underbrace{\left(\int_{t}^{t} T_{n}^{T} T_{n} T_{b} d t\right)}_{\gamma} X_{b}) d x \quad 0
\end{aligned}
$$

This corresponds to the PDE:

$$
K \sum_{i=1}^{n} \alpha_{i} \cdot X_{i} \quad D \sum_{i=1}^{n} \beta_{i} \cdot \frac{\partial^{2} X_{i}}{\partial x^{2}} \quad \gamma \cdot X_{b} \quad 0
$$

And passing all the known terms to the second member we get:

$$
K \cdot \alpha_{n} \cdot X_{n} \quad D \cdot \beta_{n} \cdot \frac{\partial^{2} X_{n}}{\partial x^{2}} \sum_{i=1}^{n}\left(K \cdot \alpha_{i} \cdot X_{i}+D \cdot \beta_{i} \cdot \frac{\partial^{2} X_{i}}{\partial x^{2}}\right)+\gamma \cdot X_{b}
$$

This equation is solved in the spatial domain with a simple finite differences or finite element method (FEM), giving the value of $X_{n}$ in the present iteration. With this value, the computation of a new value of $T_{n}$ is performed:

$$
\begin{gathered}
\int_{t}^{t}{ }_{t}^{T} T_{n} *(K \sum_{i=1}^{n} \underbrace{\left(\int_{x}^{x} X_{n}^{L} X_{i} d x\right)}_{\beta_{i}^{\prime}} \frac{\partial T_{i}}{\partial t} D \sum_{i}^{n} \underbrace{\left(\int_{x}^{x} X_{n}^{L} X_{n} \frac{\partial^{2} X_{i}}{\partial x^{2}} d x\right)}_{\alpha_{i}^{\prime}} T_{i} \\
\underbrace{\left(\int_{x 0}^{x} X_{n} X_{b} d x\right)}_{\gamma^{\prime}} T_{b}) d t \quad 0
\end{gathered}
$$

This corresponds to the PDE:

$$
K \sum_{i=1}^{n} \beta_{i}^{\prime} \cdot \frac{\partial T_{i}}{\partial t} \quad D \sum_{i=1}^{n} \alpha_{i}^{\prime} \cdot T_{i} \quad \gamma^{\prime} \cdot T_{b} \quad 0
$$

And passing all the known terms to the second member we get:

$$
K \cdot \beta_{n}^{\prime} \cdot \frac{\partial T_{n}}{\partial t} \quad D \cdot \alpha_{n}^{\prime} \cdot T_{n} \sum_{i}^{n}\left(K \cdot \beta_{i}^{\prime} \cdot \frac{\partial T_{i}}{\partial t}+D \cdot \alpha_{i}^{\prime} \cdot T_{i}\right)+\gamma^{\prime} \cdot T_{b}
$$


This equation is solved in the spatial domain with a simple implicit or Runge-Kutta method, giving the value of $T_{n}$ in the present iteration. At each iteration the new values of $X_{n}$ and $T_{n}$ are compared with the previous ones, and if the absolute value of their difference falls below a predefined threshold, the iterations are finished, and the solution is updated with the new values. This process begins again for computing the next $n+1$ mode. When the absolute value of the new mode falls below a predefined threshold, the solution is considered valid and the process finishes.

\section{Non-homogeneous boundary conditions}

To obtain the MVP in the slot, the non-homogeneous boundary conditions at the bottom of the slot (7) must be satisfied. This kind of conditions can be imposed by adding to the solution of equation (13) a new function $\psi$, continuous in $\Omega$, that verifies them (Gonzalez et al., 2010). So, the solution of equation (13) can be obtained in the form $u \quad \psi+z$, and the problem becomes:

$$
\left\{\begin{array}{lllll}
K \cdot \frac{\partial z(x, t)}{\partial t} \quad D \cdot \frac{\partial^{2} z(x, t)}{\partial x^{2}} & b(x, t) & K \cdot \frac{\partial \psi(x, t)}{\partial t} & D \cdot \frac{\partial^{2} \psi(x, t)}{\partial x^{2}} & \text { in } \Omega \quad(0, \mathrm{~L}), \mathrm{I} \quad(0, \mathrm{~T}) \\
z(0, t) & z(L, t) & 0 & \mathrm{EBC} & \text { IC } \\
z(x, 0) & u(x, 0) & \psi(x, 0) &
\end{array}\right.
$$

The problem (24) gets simplified if function $\psi$ is chosen such as:

$$
\frac{\partial^{2} \psi(x, t)}{\partial x^{2}} \quad 0 \quad \text { in } \Omega \quad(0, \mathrm{~L}), \mathrm{I} \quad(0, \mathrm{~T})
$$

In 1D, this goal can be accomplished by defining a linear function such as:

$$
\psi(x, t) \quad u(0, t)+\left(\frac{u(L, t) \quad u(0, t)}{L}\right) x \quad x \in[0, L]
$$

\section{Current in the slot solved with the separation of variables method}

In the case of the slot of Figure 1, the method of separation of variables converges in 16 modes, as shown in Figure 4.

The solution obtained by the proposed method is shown in Figure 5. After a short transient, the current reaches its steady state value, equal to the one obtained analytically (Figure 3). The norm of the relative error with respect to the theoretical solution (12), computed at the last time interval, has been shown in Figure 6 .

\section{Extension to higher dimensions}

The proposed method can be easily extended to higher dimensions: instead of solving the spatial equations in a 2D or 3D grid, the unknown field can be expressed as a product of one-dimensional modes in the form: 

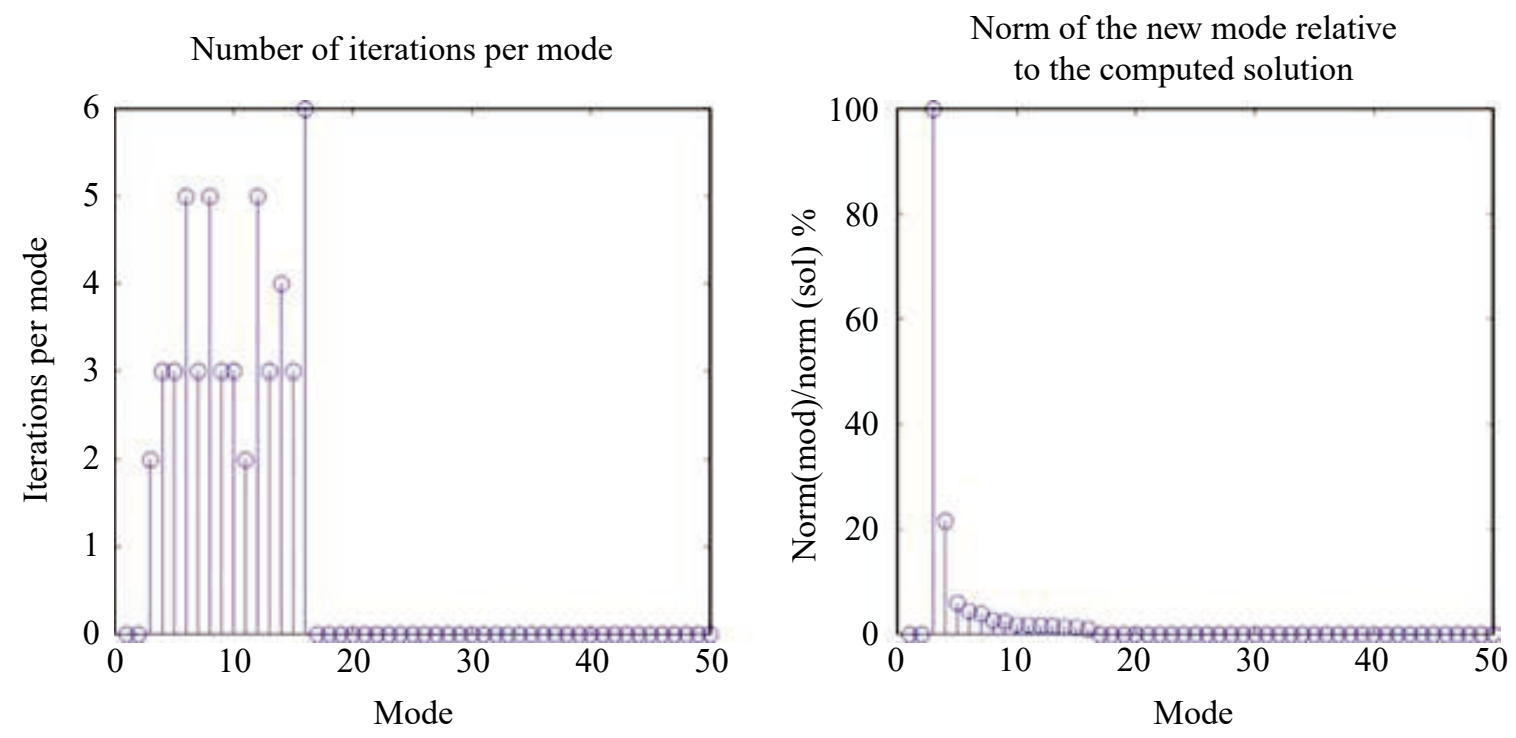

Figure 4. Number of iterations per mode (left) and norm of each mode (right)

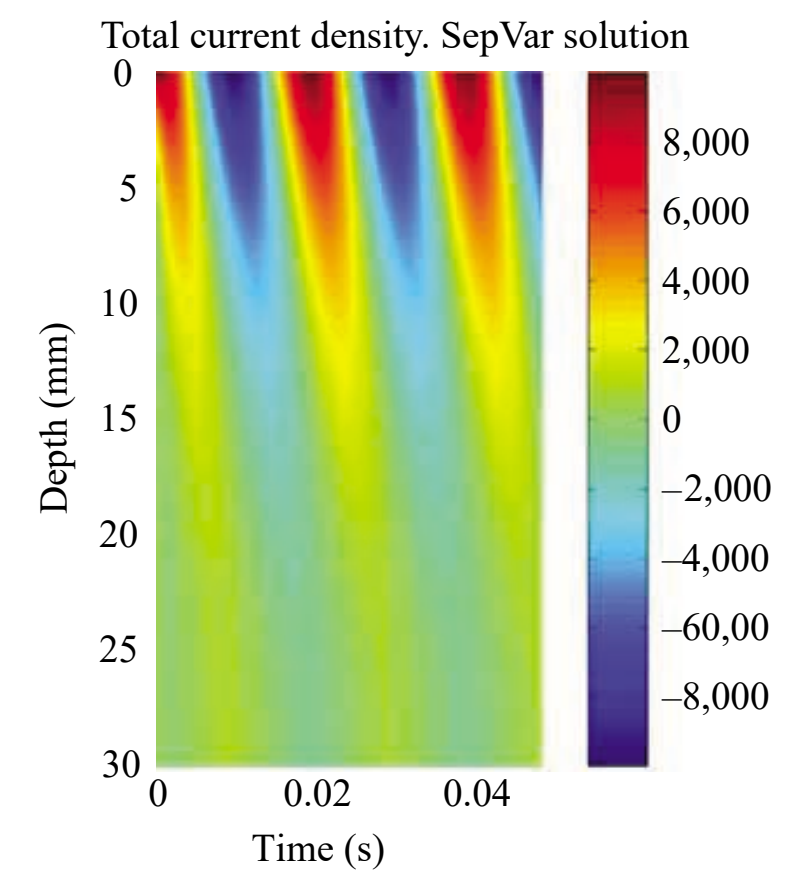

Total current density. SepVar solution

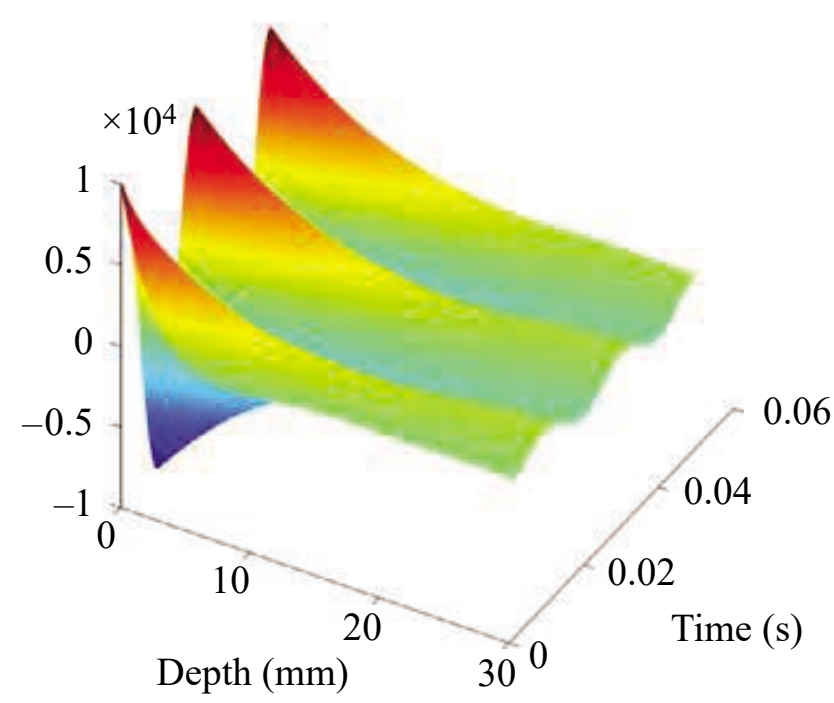

Figure 5. Total current in the slot: 2D view (left) and 3D view (right)

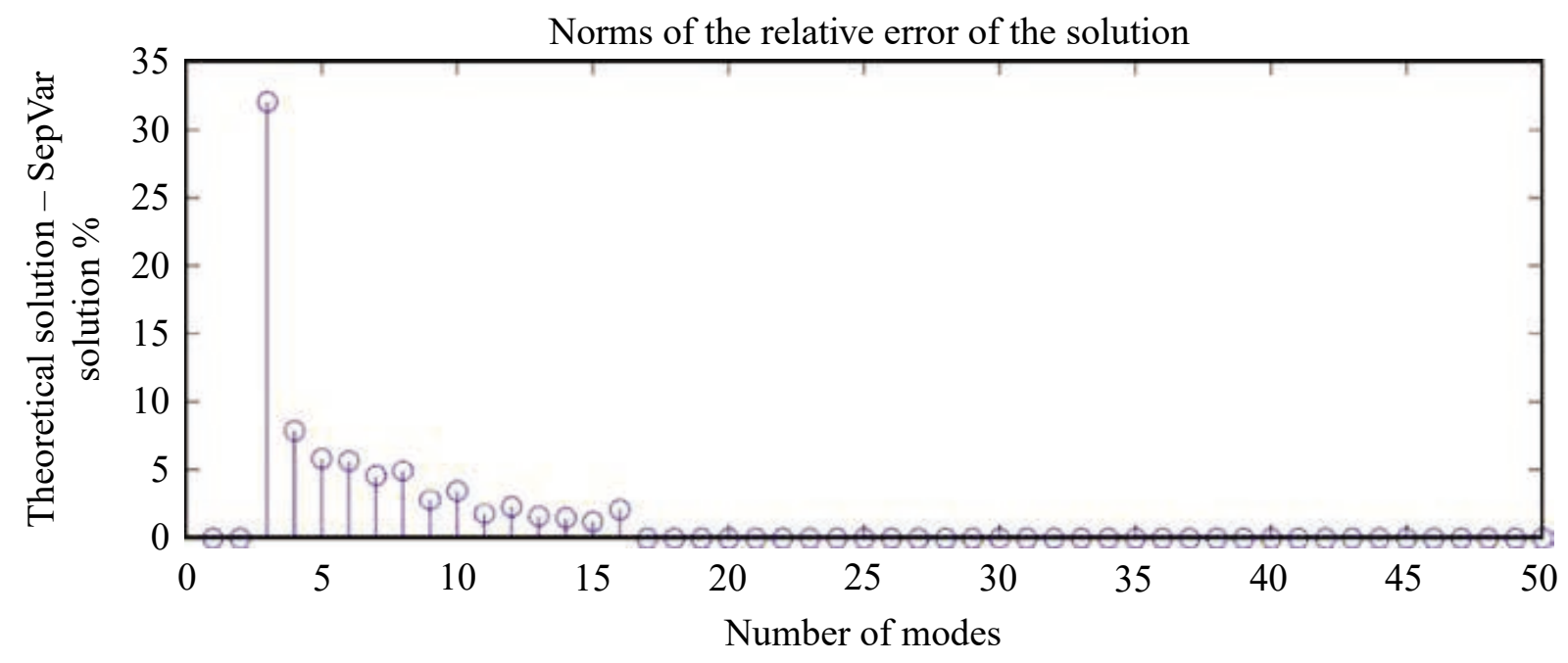

Figure 6. Difference between the theoretical solution and the solution found by the variables separation method at the last time interval 


$$
u(x, y, z, t) \quad \sum_{i=1}^{n} X_{i}(x) Y_{i}(y) Z_{i}(z) T_{i}(t)
$$

One of the difficulties of applying this technique is the imposition of $\mathrm{EBCs}$ on arbitrarily-shaped spatial domains $\Omega$. For example, a slot defined on $\mathrm{R}^{2}$ can have a shape like that in Figure 7, left. A work in progress is to treat these general domains with the method of the characteristic function (Rvachev and Sheiko, 1995). The field $u$ is multiplied by a real function $\Phi$ that acts as a "distance" function: it has continuous derivatives, and it is zero on the boundary and outside the irregular shape surface or volume of the slot. In Figure 6, right, such a function is shown for a slot in 2D. In this case, the method of variables separation is applied to the function:

$$
u(x, y, t) \quad \Phi \cdot \sum_{i=1}^{n} X_{i}(x) Y_{i}(y) T_{i}(t)
$$

\section{Conclusions}

In this paper, the method of separation of variables has been presented, and has been applied to solve the currents distribution in an electrical motor slot in transient regime. The numerical technique presented in this paper allows the numerical construction of the low dimensional functions whose product approximates the MVP in the slot. This approximation has been compared with the analytical solution to verify the accuracy of the method. The great advantage of this method is that it scales linearly with an increase in the dimensionality of the problem, instead of exponentially, as traditional mesh-based methods. Nevertheless, some issues like the application of the method to non-rectangular domains in high dimensions are an open research field.

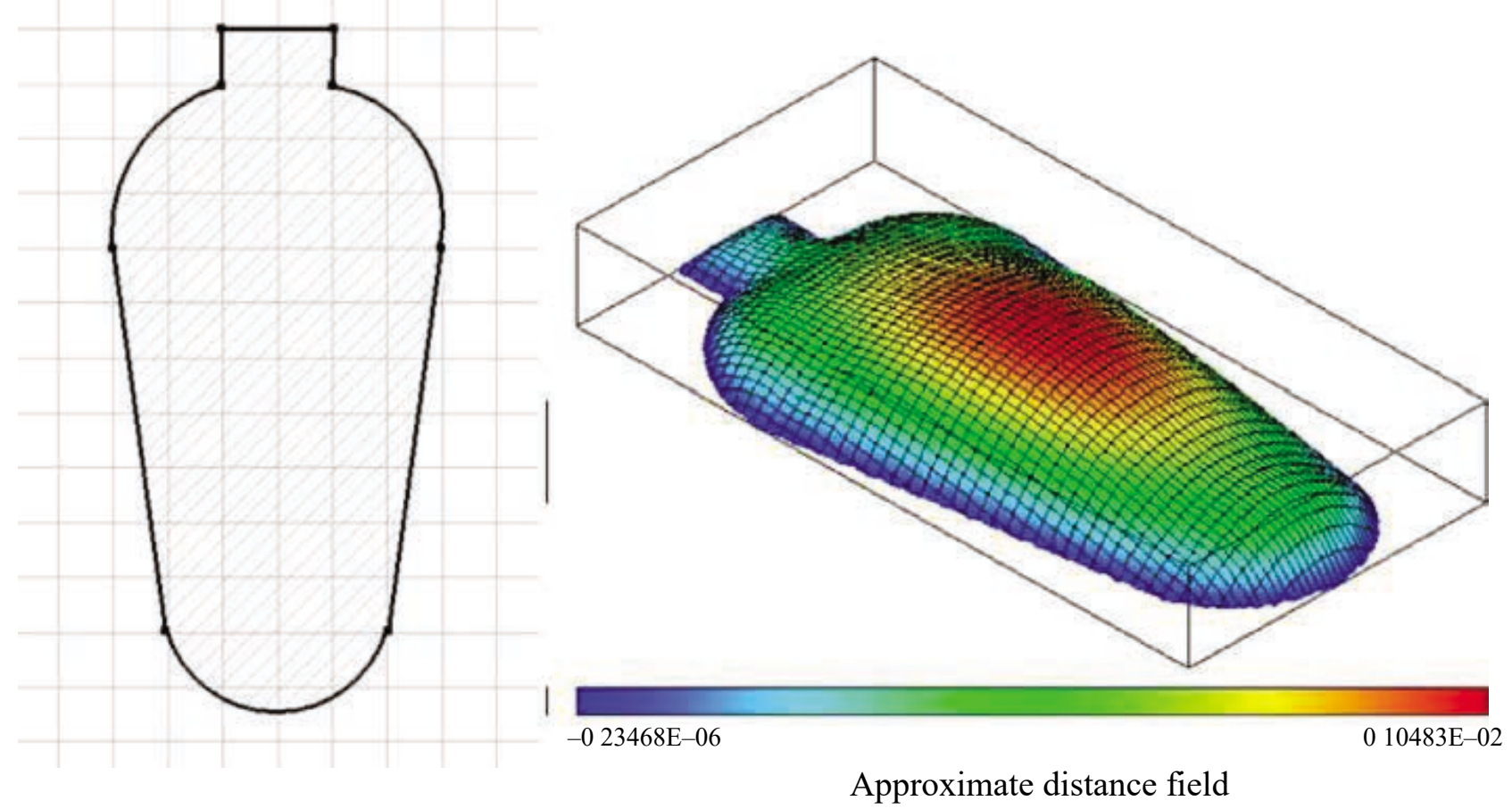

Figure 7. Slot with a complex 2D shape (left) and distance like characteristic function $\Phi$ associated to it (right) 


\section{References}

Ammar, A., Mokdad, B., Chinesta, F. and Keunings, R. (2006), "A new family of solvers for some classes of multidimensional partial differential equations encountered in kinetic theory modeling of complex fluids", Journal of Non Newtonian Fluid Mechanics, Vol. 139, pp. 15376.

Ammar, A., Mokdad, B., Chinesta, F. and Keunings, R. (2007), "A new family of solvers for some classes of multidimensional partial differential equations encountered in kinetic theory modeling of complex fluids. Part II: transient simulation using space time separated representation", Journal of Non Newtonian Fluid Mechanics, Vol. 144, pp. 9812.

Ammar, A., Normandin, M., Daim, F., Gonzalez, D., Cueto, E. and Chinesta, F. (n.d.), "Non incremental strategies based on separated representations: applications in computational rheology", Communications in Mathematical Sciences (in press).

Chinesta, F., Ammar, A., Lemarchand, F., Beauchene, P. and Boust, F. (2008), "Alleviating mesh constraints: model reduction, parallel time integration and high resolution homogenization", Computer Methods in Applied Mechanics and Engineering, Vol. 197, pp. 40013.

Gonzalez, D., Ammar, A., Chinesta, F. and Cueto, E. (2010), "Recent advances in the use of separated representations", International Journal for Numerical Methods in Engineering, Vol. 81 No. 5, pp. 63759.

Rvachev, V.L. and Sheiko, T.I. (1995), "R functions in boundary value problems in mechanics", Applied Mechanics Reviews, Vol. 48, p. 151.

\section{About the authors}

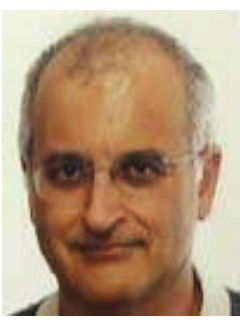

M. Pineda Sanchez was born in 1962 in Albacete (Spain). He received his Dipl. Ing. and Dr Ing. degrees in electrical engineering from the Universidad Politécnica de Valencia (Spain) in 1985 and 2004, respectively. He joined the faculty of the Universidad Politécnica de Valencia in 1987 as Professor in the Department of Electrical Engineering, in the area of theory and control of electrical machines. His research interests include electrical machines and drives, induction motor diagnostics, numerical simulation of electromagnetic fields, and software development. M. Pineda Sanchez is the corresponding author and can be contacted at: mpineda@die.upv.es

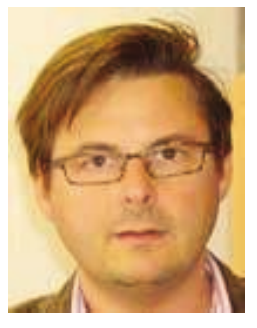

F. Chinesta, born in 1966 in Valencia (Spain), is currently Professor of Computational Mechanics at the Ecole Centrale of Nantes (France) and titular of the EADS Corporate Foundation International Chair on Advanced Modeling of Composites Manufacturing Processes. His main research contribution concerns the proposal and development of efficient model reduction strategies based on the proper orthogonal decomposition and more particularly the development of separated representations based strategies making possible the solution of models suffering the so called "curse of dimensionality". F. Chinesta is author of more than 500 scientific works, with more than 140 papers in peer review journals (120 in international journals), 31 contributions to research books and more than 300 contributions to conference proceedings.

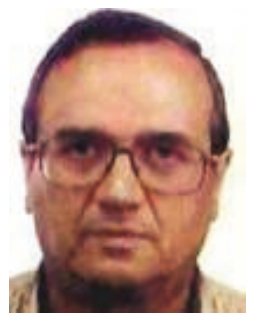

J. Roger Folch obtained his MSc degree in electrical engineering in 1970 from the Universidad Politécnica de Cataluña and his $\mathrm{PhD}$ in 1980 from the Universidad Politécnica de Valencia, Spain. From 1971 to 1978 he worked in the electrical industry as Project Engineer. He joined the Universidad Politécnica de Valencia in 1978 and is currently Professor of Electrical Installations and Machines. His main research areas are numerical methods (FEM and others) applied to the design and maintenance of electrical machines and equipment. 


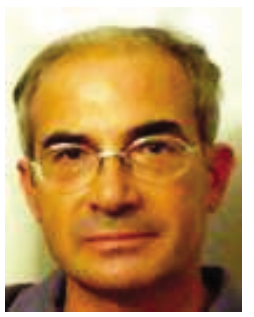

M. Riera Guasp received an MSc degree in industrial engineering and the $\mathrm{PhD}$ degree in electrical engineering from the Universidad Politécnica de Valencia (Spain) in 1981 and 1987, respectively. Currently he is an Associate Professor in the Department of Electrical Engineering of the Universidad Politécnica de Valencia. His research interests include condition monitoring of electrical machines, applications of the wavelet theory to electrical engineering, and efficiency in electric power applications.

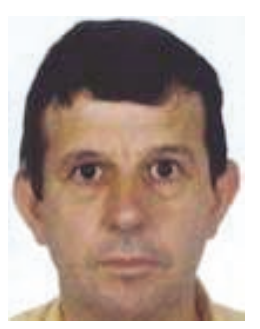

J. Pérez Cruz obtained his MSc in electrical engineering in 1997 from the Universidad Politécnica de Valencia and his $\mathrm{PhD}$ in 2006 from the same university. From 1970 to 1992 he worked in the electrical industry as an electrical technician in the field of industrial systems maintenance and automation. In 1992, he joined the Universidad Politécnica de Valencia and is currently Professor of Electrical Installations and Machines. His research interests focus on induction motor diagnostics and maintenance, numerical modelling, and automation of industrial installations.

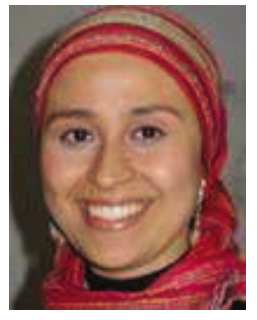

F. Darm is a postdoctorant in Centre des Matériaux of Ecole Nationale Supérieure des Mines de Paris. She has a $\mathrm{PhD}$ degree at Applied Mathematics from Laboratoire de Mathématiques d'Orsay (Paris Sud University, France) on "Theorical and numerical study in coupling between multiphase flow and mechanical deformations in hydrocarbons extraction". She is experienced in partial differential equations and algorithms of resolution, reduced order models, proper orthogonal decomposition, separation of variables method, parallel computations. 